\title{
A Novel Traveling-wave-based Protection Scheme for LCC-HVDC Systems Using Teager Energy Operator
}

\author{
Wang $\mathrm{Hao}^{\mathrm{a}}$, Sohrab Mirsaeidi ${ }^{\mathrm{b}}$, Xiaoning Kanga,*, Xinzhou Dong ${ }^{\mathrm{b}}$, Dimitrios \\ Tzelepis $^{\mathrm{c}}$ \\ ${ }^{a}$ Shaanxi Key Laboratory of Smart Grid, Department of Electrical Engineering, \\ Xi'an Jiaotong University, Shaanxi, Peoples' Republic of China \\ ${ }^{b}$ Department of Electrical Engineering, \\ Tsinghua University, Beijing, People's Republic of China \\ ${ }^{c}$ Department of Electronic 83 Electrical Engineering, \\ Strathclyde University, Glasgow, United Kingdom
}

\begin{abstract}
Line Commutated Converter (LCC) based High-Voltage Direct Current (HVDC) technology has been in operation with a high level reliability and little maintenance requirements for more than thirty years. The current-source based or classical LCC-HVDC systems are being considered for buried cable transmission as well as overhead transmission. The fault analysis and protection of LCCHVDC system is a very important aspect in terms of power system stability. This paper proposes a novel protection scheme for LCC-HVDC systems, in which the difference between propagation processes of traveling wave under internal and external faults is used as a criterion for detection of fault incidents in HVDC transmission lines. In order to quantify and intensify this difference, Teager Energy Operator (TEO) is used which has the ability to reflect the instantaneous energy of a signal. The main feature of the proposed scheme in comparison with the existing ones is that it operates faster, since it does not require to extract any harmonic or high frequency component; moreover, a 2-ms sampling window is sufficient for its algorithm which only deals with simple calculations. In order to validate the effectiveness of the proposed protection scheme, several fault events under different fault resistances and fault locations were simulated on
\end{abstract}

\footnotetext{
${ }^{*}$ Corresponding author

Email address: xiaoningkang641@gmail.com (Xiaoning Kang)
} 
a test network using PSCAD/EMTDC software. Also, the performance of the proposed scheme under real fault events was tested using four field data cases. Both simulation data and field data test results indicated that the proposed protection strategy has the ability to accurately discriminate between internal and external faults and detect the faulted pole in the bipolar systems even under high-impedance fault conditions.

Keywords: HVDC transmission line, traveling wave protection, Teager energy operator.

\section{Introduction}

High Voltage Direct Current (HVDC) transmission systems are extensively applied in recent years owing to the opportunity they provide to transfer large amounts of power over long distance more flexibly and with less losses compared 5 with HVAC transmission systems [1, 2, 3, 4. Due to the long transmission distance, HVDC transmission lines usually go through complex environment, and fault on transmission lines is one of the major threats for the whole transmission system [5, 6, 7, 8]. Protections for transmission lines act as very significant role in maintaining the reliability and security of the system.

Presently, the traveling-wave and voltage derivate protections are deployed as the main protection in the most of the HVDC transmission systems; also, backup protection in the HVDC systems is provided by under-voltage or currentdifferential protections [9, 10]. However, the performance of the traveling-wave, voltage derivate and under-voltage protections can be easily affected by the location and impedance of the fault [11, 12. Even though the differential protection schemes provide a more reliable protection rather than others, it has a long delay which makes it unable to quickly detect the fault events [13. Therefore, design of more reliable and faster protection schemes for HVDC transmission lines would be necessary.

In recent years, a large number of approaches have been proposed to present a suitable protection scheme for the HVDC systems. In [14, an improved current 
differential protection scheme is proposed, in which the current differential criterion is based on distributed parameter model. However, the criterion requires a large number of coefficients; in addition, the selection of the coefficients which varies in different actual projects influences the performance of this protection. In [15] another protection scheme is developed based on traveling wave protection. The developed scheme protection mitigates the influence of the fault impedance, but it requires more complicated calculations. Moreover, references [1] and [16] employ the difference between the measured transient impedance for internal and external faults to present suitable protection schemes for HVDC transmission systems; however, the accuracy of these schemes is highly dependent of the length of the sampling window. In fact, in order to guarantee the adequate accuracy, a relatively long sampling window may be needed. Deploying the boundary of transmission line is another approach to develop a protection scheme 35 for HVDC systems. Since the existence of smoothing reactor and DC filter which can attenuate some of the harmonics and high frequency transient components, the transit components under internal and external faults are different. In references [17] and [18] the harmonic-current-based boundary, and in reference [19] the energy-based boundary are applied to provide new protection schemes.

40 However, the above-mentioned schemes require wavelet transform to extract certain harmonics or high frequency components which increases the computation burden and sampling frequency; additionally, the effect of transmission distance on the harmonics and high-frequency components has not been taken into account in the schemes.

This paper proposes a novel protection scheme based on the fact that the propagation process of traveling wave under an internal fault is different from that under an external fault. In order to quantify the difference, Teager Energy Operator (TEO) is applied which has the ability to reflect the instantaneous energy of a signal. Even though the proposed protection in this paper still uses the effects of line boundary, it does not require to extract any harmonic or high frequency component. For the proposed scheme, the sampling frequency of $10 \mathrm{kHz}$ and the short sampling window of 2 -ms is sufficient to make the 
protection operates fast. Besides, it has the ability to protect HVDC lines against high-impedance and long distance faults.

The remainder of this paper is organized as follows: The principles of the proposed protection scheme is introduced in Section 2; Section 3 discusses the proposed protection scheme in detail; the test network for validation of the proposed scheme is introduced in Section 4; the simulation data and field data test results are presented in Section 5; and Section 6 concludes the paper.

\section{The principles of the proposed protection scheme}

\subsection{Traveling wave propagation process under a fault}

Figure 1 depicts a simple unipolar HVDC transmission system. As can be seen in the figure, the positive direction is defined as the power flow from the converter to transmission line, and Points $\mathrm{M}$ and $\mathrm{N}$ are the measuring points at

rectifier and inverter sides, respectively. The line boundary is also marked by a botted box and its details are shown in Figure 2, where $L_{s}$ is the smoothing reactor.

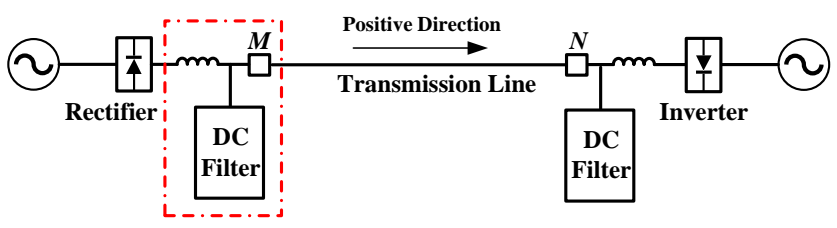

Figure 1: A simple unipolar HVDC transmission system

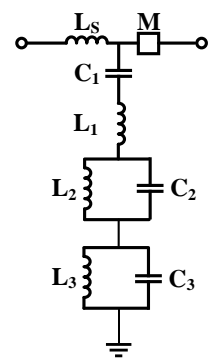

Figure 2: The structure of the line boundary 
Since the HVDC transmission system shown in Figure 1 is unipolar, the traveling wave propagation processes under internal and external faults are analyzed as follows:

The traveling wave propagation process under an internal fault is shown in Figure 3. As can be seen in the figure, when an internal fault occurs, traveling wave is generated at the fault point and propagates from fault point to two line terminals. The initial backward traveling wave which does not pass through the smoothing reactor and DC filter can be detected at point M.

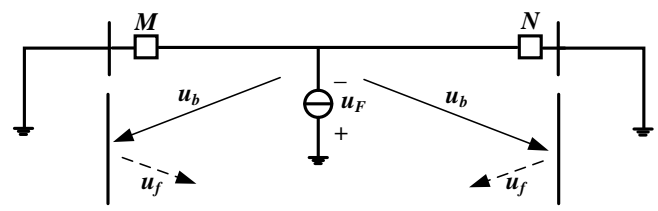

Figure 3: Traveling wave propagation process under an internal fault

When an external fault occurs at inverter side, the initial backward traveling wave can be also detected at point $\mathrm{M}$, similar to that during an internal fault with the difference that the initial backward traveling wave detected at point $\mathrm{M}$ in this case passes through the smoothing reactor and DC filter. The traveling 80 wave propagation process under an external fault at inverter side is shown in Figure 4 .

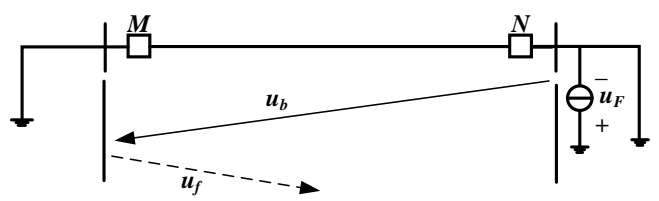

Figure 4: Traveling wave propagation process under an external fault at inverter side

Contrary to the propagation process under an internal fault or an external fault at inverter side, the initial backward traveling wave cannot be detected at point $\mathrm{M}$ under an external fault at rectifier side. The traveling wave propagation process under an external fault at rectifier side is shown in Figure 5. 


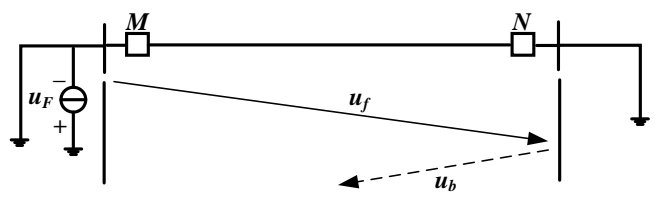

Figure 5: Traveling wave propagation process under an external fault at rectifier side

\subsection{The smoothing effects of line boundary}

In an LCC-HVDC system, a smoothing reactor and a DC filter are installed at both terminals of the transmission line and make a line boundary, as illustrated in Figure 2. Since the line boundary has an attenuation on some harmonics and high frequency components, it functions as a low-pass filter which removes the step characteristic of the signals and makes them smooth after being refracted. In other words, the signals no longer have sudden changes after passing through the ling boundary. Figure 6 demonstrates the waveforms of a step signal before and after passing through the line boundary, in which the function of line boundary can be seen visually.

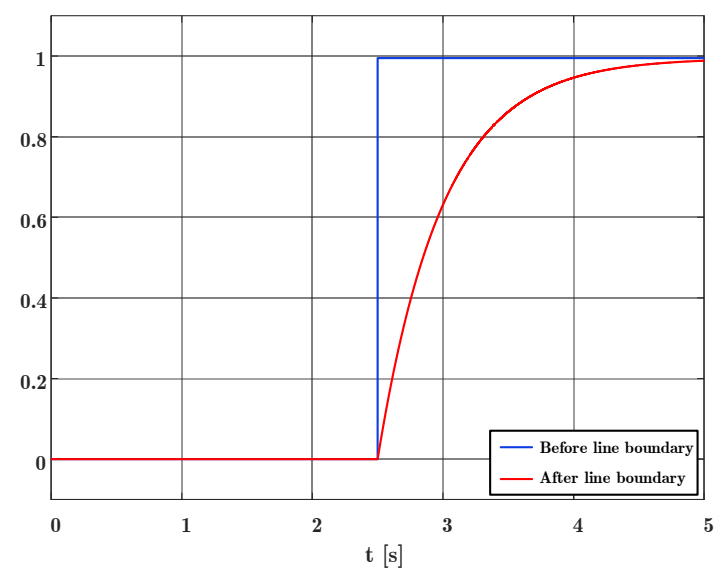

Figure 6: The waveforms of a step signal before and after passing through the line boundary 


\subsection{Teager Energy Operator}

The Teager Energy Operator (TEO) is defined as a nonlinear energy tracking signal operator which measures instantaneous energy changes of a signal and can be expressed as [20]:

$$
\psi[(x)]=[\dot{x}(t)]^{2}-x(t) \ddot{x}(t)
$$

100 waveform is smoothing and has no sudden change; If the external fault takes 
place at the rectifier side, point $\mathrm{M}$ cannot detect the initial backward traveling wave theoretically, and the waveform of traveling wave has obviously no sudden change even by considering the measurement error.

Figure 7 depicts the waveform of the backward traveling wave detected at point $\mathrm{M}$ under an internal fault and two types of external faults. The sudden change in the waveform of backward traveling wave under the internal fault can be considered as a criterion to discriminate it from the external faults either in the rectifier side or in the inverter side. According to (3) in Subsection 2.3, TEO not only has the ability to quantify the difference between the waveforms, but it can also intensify the difference to facilitate the discrimination between internal and external faults.

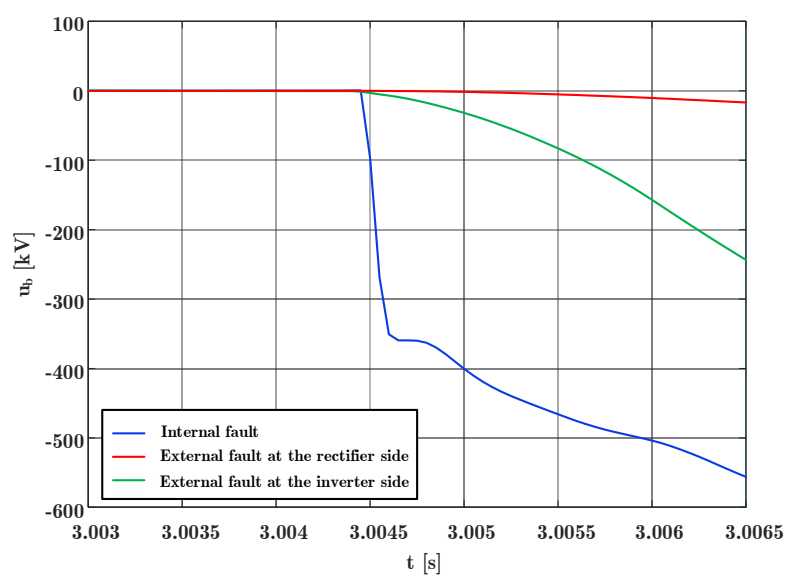

Figure 7: Waveform of the backward traveling wave under an internal fault and two types of external faults

\section{Proposed protection scheme}

3.1. Calculations of backward traveling wave and TEO

For a unipolar transmission system, the backward traveling wave is:

$$
u_{b}=\frac{\Delta u-z_{c} \Delta i}{2}
$$


Where $u_{b}$ is the traveling wave, $z_{c}$ is the surge impedance of transmission line, and $\Delta u$ and $\Delta i$ are fault components of the measured voltage and current at the rectifier side, respectively.

In a bipolar transmission system, the proposed protection is applied to each of two poles. The line-mode component of backward traveling wave is used and can be expressed as:

$$
u_{b}=\frac{\Delta u_{1}-z_{c} \Delta i_{1}}{2}
$$

where subscript 1 represents the line-mode components, and $\Delta u_{1}$ and $\Delta i_{1}$ are calculated by:

$$
\begin{gathered}
\Delta u_{1}=\frac{\sqrt{2}}{2}\left(\Delta u_{p}-\Delta u_{n}\right) \\
\Delta i_{1}=\frac{\sqrt{2}}{2}\left(\Delta i_{p}-\Delta i_{n}\right)
\end{gathered}
$$

where subscripts $p$ and $n$ represent the positive and negative poles, respectively.

Defining $S(t)$ as the TEO of the backward traveling wave:

$$
S(t)=\psi\left[u_{b}(t)\right]
$$

Substituting (8) into (1):

$$
S(t)=\dot{u}_{b}^{2}(t)-u_{b}(t) \ddot{u}_{b}(t)
$$

Considering the sampling signal is discrete, the discrete form of $S(t)$ is expressed as:

$$
S(k \Delta t)=\dot{u}_{b}^{2}(k \Delta t)-u_{b}(k \Delta t) \ddot{u}_{b}(k \Delta t)
$$




$$
\begin{gathered}
\dot{u}_{b}^{2}(k \Delta t)=u_{b}[(k+1) \Delta t]-u_{b}(k \Delta t) \\
\ddot{u}_{b}^{2}(k \Delta t)=u_{b}[(k+2) \Delta t]-2 u_{b}[(k+1) \Delta t]+u_{b}(k \Delta t)
\end{gathered}
$$

where, $k=0,1,2, \ldots, t$ is the sampling interval.

\subsection{Fault discrimination criterion}

As discussed in Section 2, the sudden change in the waveform of backward traveling wave under the internal fault can be considered as a criterion to discriminate it from the external faults either in the rectifier side or in the inverter side. In this paper, $S(t)$ is used to not only quantify the difference between the waveforms, but also to intensify the difference to facilitate the discrimination between internal and external faults. And it is calculated by the sampling data of 2-ms sampling windows, from $1 \mathrm{~ms}$ before the trigger instant to $1 \mathrm{~ms}$ after the trigger instant. Since the energy represented by TEO of backward traveling wave is concentrated in a small area around the sudden change under an internal fault, only a 0.4 -ms sampling window is enough for internal fault cases. $S_{T E O}$ is defined as:

$$
S_{T E O}=\int_{t_{0}-t_{1}}^{t_{0}+t_{1}} S(t) \mathrm{d} t
$$

where $t_{0}$ is the trigger moment of starting unit and $t_{1}=0.2 \mathrm{~ms}$. Its discrete form is expressed as:

$$
S_{T E O}=\sum_{k=-N_{1}}^{N_{1}} S\left(t_{0}+k \Delta t\right) \Delta t
$$

where $N_{1}$ is the number of sampling points in $0.2 \mathrm{~ms}$. For the protection algorithm, the multiplication by $\Delta t$ in (14) is not necessary, and can be neglected 
to reduce the computations. So, $S_{T E O}$ is rewritten as:

$$
S_{T E O}=\sum_{k=-N_{1}}^{N_{1}} S\left(t_{0}+k \Delta t\right)
$$

The discrimination criterion can be defined as:

$$
S_{T E O}>S_{\text {set }}
$$

where $S_{\text {set }}$ is the threshold which is set according to the maximum $S_{T E O}$ under external faults. If (16) is satisfied, the fault is judged as an internal fault, and otherwise as an external fault. It should be noted that the proposed criterion may be affected by the lightening disturbances. The lightning disturbances can be of two types, i.e. induction lightning disturbances and direct lightning strokes. During induction lightning disturbances, both positive and negative lines are affected. The protection algorithm uses the line-mode component of the backward traveling wave which is calculated by Equations (5) to (7), and hence, the effect of lightning disturbances can be offset; but direct lightning strokes can negatively influence the criterion. So, a lightning identification element should be added to ensure the reliability of the protection.

\subsection{Criterion for selection of the faulted pole}

In a bipolar HVDC transmission system, it is of considerable importance to select and isolate the faulted pole correctly so that the healthy pole continues operating. According to 21], the polarity of the earth-mode of backward traveling wave $u_{b 0}$ is negative under a Line-to-Ground (LG) fault event on the positive pole, whereas it is positive under an LG fault event on the negative pole. Also, in case a Line-to-Line (LL) fault occurs, $u_{b 0}$ is theoretically equal to zero, and $u_{b 0}$ can be calculated by:

$$
u_{b 0}=\frac{\Delta u_{0}-z_{c 0} \Delta i_{0}}{2}
$$




$$
\begin{gathered}
\Delta u_{0}=\frac{\sqrt{2}}{2}\left(\Delta u_{p}+\Delta u_{n}\right) \\
\Delta i_{0}=\frac{\sqrt{2}}{2}\left(\Delta i_{p}+\Delta i_{n}\right)
\end{gathered}
$$

where, $z_{c 0}$ is the earth-mode component of the surge impedance of the transmission line.

According to the above-mentioned analysis, the value of $u_{b 0}\left(t_{0}+N \Delta t\right)$ is

The criterion is defined as follows:

(a) If $u_{b 0}\left(t_{1}+N \Delta t\right)<-0.5$, the fault is of LG type and has occurred on the positive pole.

(b) If $u_{b 0}\left(t_{1}+N \Delta t\right)>0.5$, the fault is of LG type and has occurred on the negative pole.

(c) If $-0.5<u_{b 0}\left(t_{1}+N \Delta t\right)<0.5$, the fault is of LL type.

\subsection{Flow chart of the proposed protection scheme}

Figure 8 demonstrates the flow chart of the proposed protection scheme which is applied as the main protection for the HVDC transmission line. As can be seen in the figure, the data of voltage and current are obtained in the rectifier side, and tested by a starting unit which can be expressed as:

$$
\left\{\begin{array}{l}
\left|\Delta u_{p}\right|>\left|\Delta u_{p}\right|_{\text {threshold }} \\
\text { or } \\
\left|\Delta u_{n}\right|>\left|\Delta u_{n}\right|_{\text {threshold }}
\end{array}\right.
$$

where $\left|\Delta u_{p}\right|_{\text {threshold }}$ and $\left|\Delta u_{n}\right|_{\text {threshold }}$ are set to $5 \%$ of the rated DC voltage.

If (20) is satisfied, $S_{T E O}$ and $u_{b 0}$ are calculated, and then if $S_{T E O}>S s e t$ an internal fault is detected. Finally, the criterion for selection of the faulted 


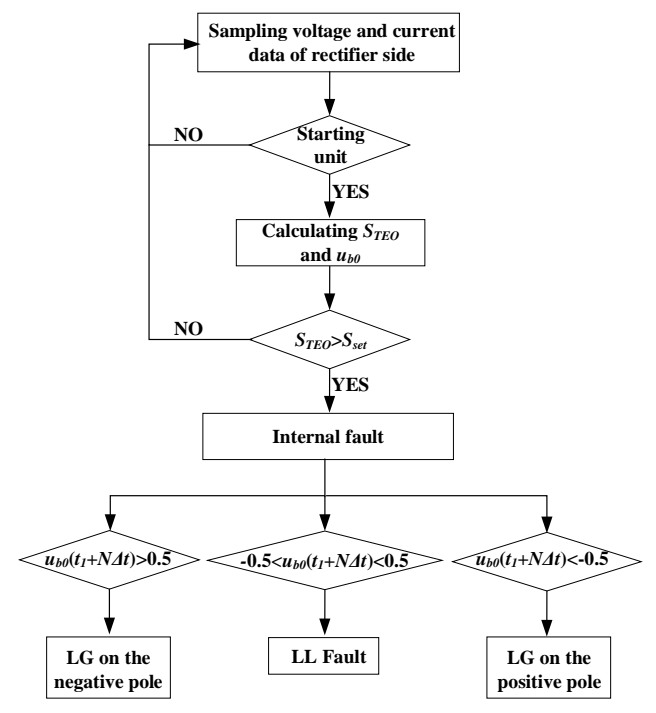

Figure 8: Flow chart of the proposed protection scheme

\section{Test Network}

In order to validate the effectiveness of the proposed control strategy in this paper, several simulations have been performed using PSCAD/EMTDC software. The single-line diagram of a bipolar $500 \mathrm{kV}$ HVDC transmission system along with its tower structure which is used as test network in this paper is depicted in Figure 9. The test network which is simulated based on Deyang-Baoji $\pm 500 \mathrm{kV}$ HVDC transmission system in China has a length of $534 \mathrm{~km}$ and the transmission capacity of $3000 \mathrm{MW}$. The detailed parameters of the test network and its DC filter with the same structure of that shown in Figure 2 are listed in Tables 1 and 2.

Table 1: Detailed parameters of the test network

\begin{tabular}{ccc}
\hline \hline Parameter [Unit] & Rectifier side & Inverter side \\
\hline Line-to-Line AC voltage $[\mathrm{kV}]$ & 345 & 230 \\
Equivalent impedance $[\Omega]$ & $2.53 \angle 84$ & $2.05 \angle 75$ \\
DC filter & Triple tuned & Triple tuned \\
Smoothing reactor $[\mathrm{mH}]$ & 150 & 150 \\
\hline \hline
\end{tabular}




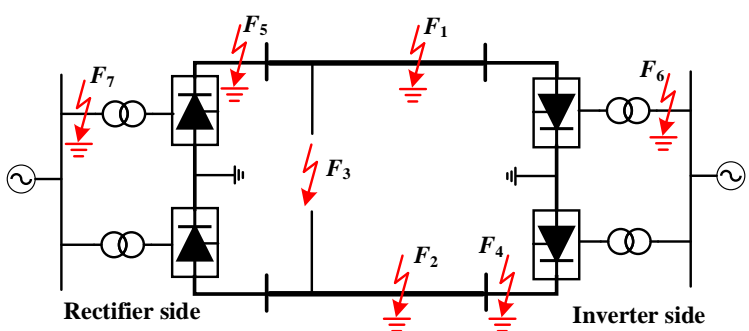

(a)
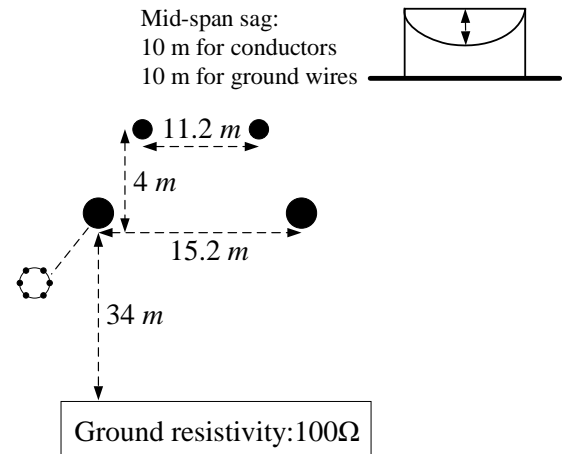

(b)

Figure 9: Test network (a) The single-line diagram (b) Structure of DC tower

Table 2: Detailed parameters of the test network

\begin{tabular}{cc}
\hline \hline Parameter [Unit] & Value \\
\hline$C_{1}[\mu \mathrm{F}]$ & 4 \\
$C_{2}[\mu \mathrm{F}]$ & 11.198 \\
$C_{3}[\mu \mathrm{F}]$ & 15.52 \\
$L_{1}[\mathrm{mH}]$ & 4.374 \\
$L_{2}[\mathrm{mH}]$ & 4.153 \\
$L_{3}[\mathrm{mH}]$ & 0.842 \\
\hline \hline
\end{tabular}




\section{Simulation data and field data test results}

\subsection{Simulation data test results}

\subsection{Field data test results}

Even though PSCAD/EMTDC is a powerful software in electromagnetic 225

In order to validate the effectiveness of the proposed protection scheme, several fault events under different fault resistances and fault locations were simulated on the test network, as shown in Figure 9(a). For the internal faults, the fault distance is defined as the distance from the fault point to the measurement point at the rectifier; also, $S_{\text {set }}$ and the sampling frequency are set to 5000 and $10 \mathrm{kHz}$.

In order to illustrate the performance of the proposed protection scheme, the simulation results for internal fault F1 with the fault distance of $250 \mathrm{~km}$ and fault resistance of $1 \Omega$, and external fault F4 are shown in Figures 10 and 11, respectively. In both cases, the sampling window is set to $2 \mathrm{~ms}$, from $1 \mathrm{~ms}$ before the trigger instant to $1 \mathrm{~ms}$ after the trigger instant. For internal fault F1, $S_{T E O}$ has been calculated from Figure10(a) and equals 209850; also, it can be seen from Figure 10(b) that the polarity of earth-mode of backward traveling wave is negative. Hence, the protection algorithm judges that the fault is LG internal and has occurred on the positive pole. Similarly, $S_{T E O}$ for external fault F4 can be calculated from Figure 11 which is equivalent to 408, and hence, the protection algorithm judges that the fault is external in this case.

Tables 3 and 4 list the simulation data test results of the proposed protection scheme under different internal and external fault conditions. From the tables, it can be seen that the proposed protection is able to correctly discriminate different faults even under long-distance faults or high resistance faults.

\footnotetext{
transient simulations, it is unable to accurately simulate the real scenarios in
} 


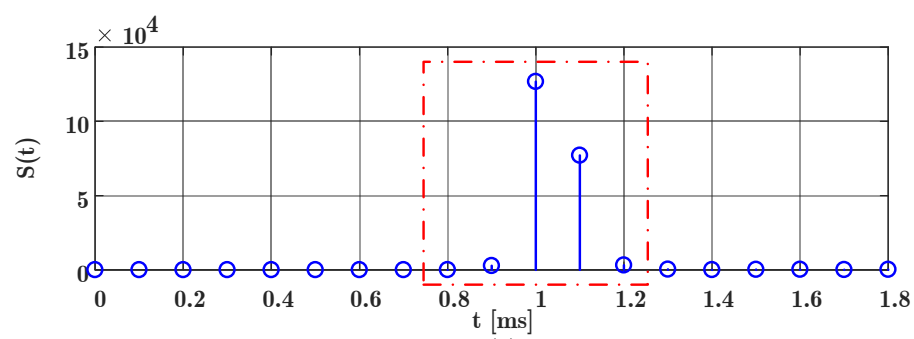

(a)

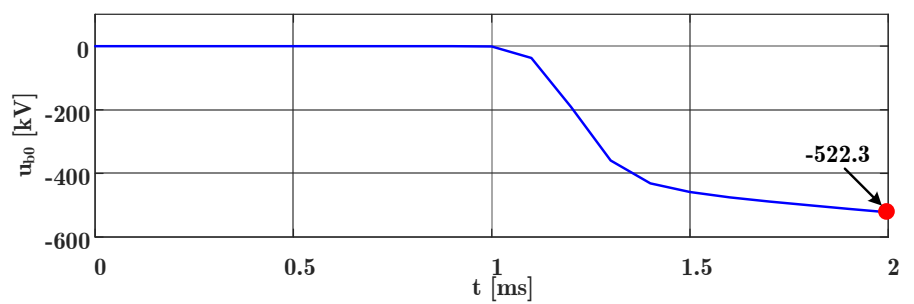

(b)

Figure 10: Simulation results for internal fault F1 with the fault distance of $250 \mathrm{~km}$ and fault resistance of $1 \Omega$. (a) $S(t)$ of F1. (b) Waveform of $u_{b 0}$

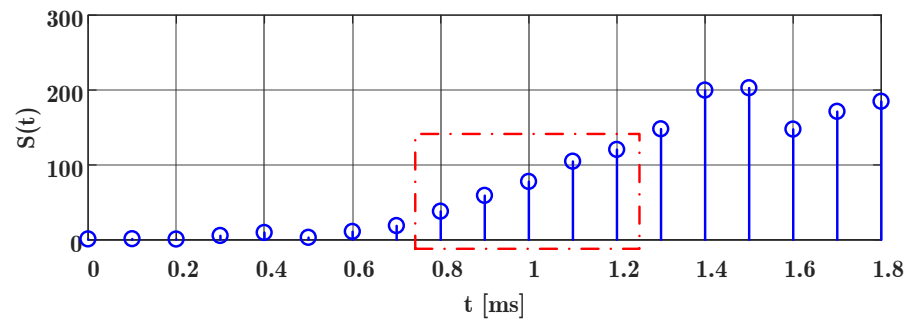

Figure 11: Simulation results for external fault F4

power systems. Thus, in order to test the performance of the proposed protection scheme under real fault events, field data is also used in this paper.

All the field data used in this paper are obtained from the short-circuit tests on Lingzhou-Shaoxing $\pm 800 \mathrm{kV}$ UHVDC system with the length of $1720 \mathrm{~km}$. Figure 12 shows the voltage and current waveforms of four field data cases. The test results of the proposed protection scheme for these cases are listed in Table 5. Case 1 is an internal fault close to the rectifier (Lingzhou) on the positive pole; Case 2 is an internal fault close to the inverter (Shaoxing) on the positive pole; 
Table 3: Simulation data test results of the proposed protection scheme under different internal fault conditions

\begin{tabular}{cccc}
\hline \hline Applied Fault & $S_{T E O}$ & $u_{b 0}[\mathrm{kV}]$ & Result \\
\hline $\mathrm{F} 1,1 \mathrm{~km}, 1 \Omega$ & 8573700 & -323.3 & LG Internal Fault on Positive Pole \\
$\mathrm{F} 1,0 \mathrm{~km}, 200 \Omega$ & 165180 & -321 & LG Internal Fault on Positive Pole \\
$\mathrm{F} 1,0 \mathrm{~km}, 500 \Omega$ & 25677 & -165.3 & LG Internal Fault on Positive Pole \\
$\mathrm{F} 1,250 \mathrm{~km}, 1 \Omega$ & 209850 & -522.3 & LG Internal Fault on Positive Pole \\
$\mathrm{F} 1,250 \mathrm{~km}, 200 \Omega$ & 40173 & -234.8 & LG Internal Fault on Positive Pole \\
$\mathrm{F} 1,250 \mathrm{~km}, 500 \Omega$ & 17561 & -132.1 & LG Internal Fault on Positive Pole \\
$\mathrm{F} 1,500 \mathrm{~km}, 1 \Omega$ & 152440 & -397.2 & LG Internal Fault on Positive Pole \\
$\mathrm{F} 1,500 \mathrm{~km}, 200 \Omega$ & 30798 & -161.3 & LG Internal Fault on Positive Pole \\
$\mathrm{F} 1,500 \mathrm{~km}, 500 \Omega$ & 10783 & -70.3 & LG Internal Fault on Positive Pole \\
$\mathrm{F} 2,0 \mathrm{~km}, 200 \Omega$ & 164690 & 322.7 & LG Internal Fault on Negative Pole \\
$\mathrm{F} 3,500 \mathrm{~km}, 200 \Omega$ & 332740 & 0.08 & LL Internal Fault \\
\hline \hline
\end{tabular}

Table 4: Simulation data test results of the proposed protection scheme under different external fault conditions

\begin{tabular}{ccc}
\hline \hline Applied Fault & $S_{T E O}$ & Result \\
\hline F4 & 408 & External Fault \\
F4 & 0.5 & External Fault \\
F5 & 1993 & External Fault \\
F6, 1ph & 412 & External Fault \\
F6, 3ph & 0.4 & External Fault \\
F7, 1ph & 1.3 & External Fault \\
F7, 3ph & 408 & External Fault \\
\hline \hline
\end{tabular}

Case 3 is an internal fault close to the inverter (Shaoxing) on the negative pole; and, Case 4 is an AC external fault under $50 \%$ of the voltage level $( \pm 400 \mathrm{kV})$. As can be seen from Table 5, the proposed protection algorithm has a correct performance in all of the field data cases and has the ability to discriminate between internal and external faults; hence, the proposed scheme can be effective in protection of real HVDC transmission systems against different types of faults.

Table 5: Test results of the proposed protection scheme for four field data cases

\begin{tabular}{cccc}
\hline \hline Applied Fault & $S_{T E O}$ & $u_{b 0}[\mathrm{kV}]$ & Result \\
\hline Case 1 & 42475 & -969.6 & LG Internal Fault on Positive Pole \\
Case 2 & 38369 & -264.4 & LG Internal Fault on Positive Pole \\
Case 3 & 63317 & 195.4 & LG Internal Fault on Negative Pole \\
Case 4 & 670 & - & External Fault \\
\hline \hline
\end{tabular}



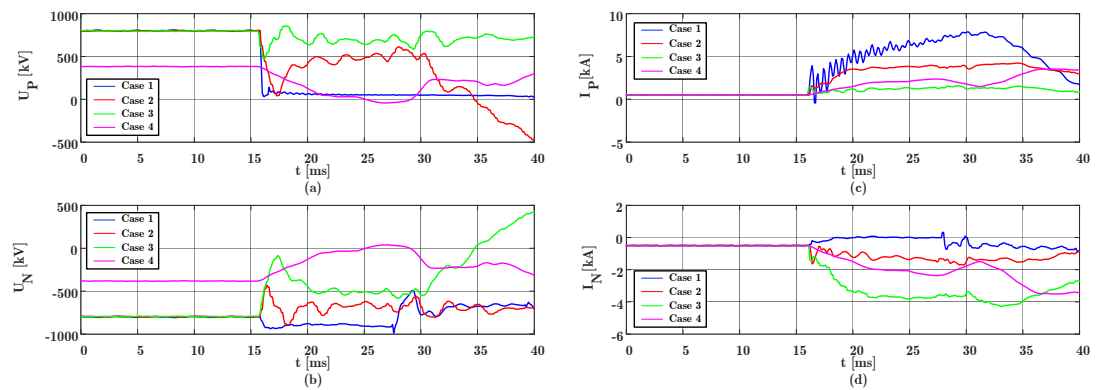

Figure 12: Voltage and current waveforms of four field data cases. (a) voltage waveform of the positive pole. (b) voltage waveform of the negative pole. (c) current waveform of the positive pole. (d) current waveform of the negative pole.

\section{Conclusion}

In this paper, a novel protection scheme was proposed based on the fact that the propagation process of traveling wave under an internal fault is different from that under an external fault. TEO was used to not only quantify this difference, but also to intensify the difference to facilitate the discrimination between internal and external faults; in addition, the polarity of the earth-mode component of backward traveling was applied to select the faulted pole when an internal fault occurs in a bipolar HVDC system. In order to verify the efficacy of the proposed scheme, several simulations were conducted using PSCAD/EMTDC software. Both simulation data and field data test results indicated the proposed algorithm has the ability to accurately discriminate between internal and external faults and protect HVDC transmission lines against different types of faults.

\section{Acknowledgment}

This work was supported in part by the National Natural Science Foundation of China (Grant No. 51120175001), and in part by the National Key Research and Development Plan of China (Grant No. 2016YFB0900600). 


\section{References}

[1] M. . Cardiel-lvarez, J. L. Rodriguez-Amenedo, S. Arnaltes, M. E. MontillaDJesus, Modeling and control of LCC rectifiers for offshore wind farms connected by HVDC links, IEEE Transactions on Energy Conversion 32 (4) (2017) 1284-1296. doi:10.1109/TEC.2017.2696261.

[2] B. Hu, K. Xie, H. M. Tai, Optimal reliability allocation of $p m$ 800kv ultra HVDC transmission systems, IEEE Transactions on Power Delivery PP (99) (2017) 1-1. doi:10.1109/TPWRD.2017.2775099.

[3] M. Barnes, D. V. Hertem, S. P. Teeuwsen, M. Callavik, HVDC systems in smart grids, Proceedings of the IEEE 105 (11) (2017) 2082-2098. doi: 10.1109/JPROC.2017.2672879.

[4] M. Farshad, J. Sadeh, A novel fault-location method for HVDC transmission lines based on similarity measure of voltage signals, IEEE Transactions on Power Delivery 28 (4) (2013) 2483-2490. doi:10.1109/TPWRD.2013. 2272436 .

[5] J. M. Johnson, A. Yadav, Complete protection scheme for fault detection, classification and location estimation in HVDC transmission lines using support vector machines, IET Science, Measurement Technology 11 (3) (2017) 279-287. doi:10.1049/iet-smt.2016.0244.

[6] F. D. Marvasti, A. Mirzaei, A novel method of combined DC and harmonic overcurrent protection for rectifier converters of monopolar HVDC Systems, IEEE Transactions on Power Delivery PP (99) (2017) 1-1. doi:10.1109/ TPWRD.2017.2745758

[7] J. Suonan, J. Zhang, Z. Jiao, L. Yang, G. Song, Distance protection for HVDC transmission lines considering frequency-dependent parameters, IEEE Transactions on Power Delivery 28 (2) (2013) 723-732. doi: 10.1109/TPWRD . 2012.2232312. 
[8] H. Xiao, Y. Li, R. Liu, X. Duan, Single-end time-domain transient electrical signals based protection principle and its efficient setting calculation method for LCC-HVDC lines, IET Generation, Transmission Distribution 11 (5) (2017) 1233-1242. doi:10.1049/iet-gtd.2016.1159.

[9] D. Naidoo, N. M. Ijumba, A protection system for long HVDC transmission lines, in: 2005 IEEE Power Engineering Society Inaugural Conference and Exposition in Africa, 2005, pp. 150-155. doi:10.1109/PESAFR.2005. 1611805 .

[10] A. Li, Z. Cai, Q. Sun, X. Li, D. Ren, Z. Yang, Study on the dynamic performance characteristics of HVDC control and protections for the HVDC line fault, in: 2009 IEEE Power Energy Society General Meeting, 2009, pp. 1-5. doi:10.1109/PES.2009.5275974.

[11] J. Liu, N. Tai, C. Fan, Y. Yang, Transient measured impedance-based protection scheme for DC line faults in ultra high-voltage direct-current system, IET Generation, Transmission Distribution 10 (14) (2016) 35973609. doi:10.1049/iet-gtd.2016.0408.

[12] F. Kong, Z. Hao, S. Zhang, B. Zhang, Development of a novel protection device for bipolar HVDC transmission lines, IEEE Transactions on Power Delivery 29 (5) (2014) 2270-2278. doi:10.1109/TPWRD.2014.2305660.

[13] S. Chakraborty, Online monitoring and protection of voltage stability for LCC based HVDC system, in: 2016 IEEE 7th Power India International Conference (PIICON), 2016, pp. 1-6. doi:10.1109/POWERI.2016.8077425.

[14] S. p. Gao, Q. Liu, G. b. Song, Current differential protection principle of HVDC transmission system, IET Generation, Transmission Distribution 11 (5) (2017) 1286-1292. doi:10.1049/iet-gtd.2016.1380

[15] J. Wu, H. Li, G. Wang, Y. Liang, An improved traveling-wave protection scheme for LCC-HVDC transmission lines, IEEE Transactions on Power Delivery 32 (1) (2017) 106-116. doi:10.1109/TPWRD.2016.2549565. 
[16] Z. Li, G. Zou, T. Du, W. Yang, S-transform based pilot protection method for HVDC transmission lines, in: 2015 5th International Conference on Electric Utility Deregulation and Restructuring and Power Technologies (DRPT), 2015, pp. 1667-1672. doi:10.1109/DRPT . 2015.7432519.

[17] Z. Zheng, T. Tai, J. S. Thorp, Y. Yang, A transient harmonic current protection scheme for HVDC transmission line, IEEE Transactions on Power Delivery 27 (4) (2012) 2278-2285. doi:10.1109/TPWRD.2012.2201509.

[18] G. Song, X. Chu, S. Gao, X. Kang, Z. Jiao, A new whole-line quickaction protection principle for HVDC transmission lines using one-end

【 current, IEEE Transactions on Power Delivery 30 (2) (2015) 599-607. doi: 10.1109/TPWRD. 2014.2300183.

[19] X. Zheng, T. Nengling, Y. Guangliang, D. Haoyin, A transient protection scheme for HVDC transmission line, IEEE Transactions on Power Delivery 27 (2) (2012) 718-724. doi:10.1109/TPWRD.2011.2179321.

[20] Q. Yang, J. Mei, J. Xiao, L. Zhang, Y. Xiao, A transient protection scheme for HVDC transmission line, Journal of vibration and shock 34 (6) (2016) $1-5$.

[21] S. Lou, X. Dong, Single-end protection of DC transmission lines based 330 on fault travelling wave process, Guangdong Electric Power 29 (9) (2016) $52-57$. 